\title{
Label-free imaging of heme proteins with two-photon excited photothermal lens microscopy
}

\author{
Sijia Lu, Wei Min, Shasha Chong, Gary R. Holtom, and X. Sunney Xie ${ }^{a)}$ \\ Department of Chemistry and Chemical Biology, Harvard University, Cambridge, \\ Massachusetts 02138, USA
}

(Received 21 November 2009; accepted 11 January 2010; published online 17 March 2010)

\begin{abstract}
Heme proteins, such as hemoglobins and cytochromes, play important roles in various biological processes. Here we employ the two-photon excited photothermal effect as a contrast mechanism to map heme proteins distribution. Particularly, both a thermal lens scheme and a high-frequency modulation are utilized to enhance the signal-to-noise ratio. We demonstrate label-free imaging of individual red blood cells, subcellular distribution of cytochromes in live mammalian cells, and the microvascular networks in mouse ear tissue and in a zebrafish gill. (c) 2010 American Institute of Physics.
\end{abstract}

[doi:10.1063/1.3308485]

Heme proteins, such as hemoglobins and cytochromes, are important biological chromophores in almost all living organisms, as they participate in crucial processes such as electron and oxygen transport and apoptosis. To be able to image the distribution of heme proteins with high sensitivity could greatly facilitate biomedical studies such as tumor angiogenesis and apoptosis signaling. ${ }^{1,2}$ In particular, medical applications such as vascular imaging require threedimensional (3D) high spatial resolution down to single capillaries and image contrast generated by endogenous means would be preferable to exogenous contrast agents. However, although heme proteins strongly absorb visible light in their Soret and $Q$ bands, they exhibit extremely weak fluorescence quantum yields $\left(<10^{-5}\right) \cdot{ }^{3,4}$ Hence, developing a label-free nonfluorescence optical imaging technique to visualize these chromophores in their natural physiological environment is both a rewarding and a challenging endeavor.

Here, we report the use of two-photon excited photothermal effect as a contrast mechanism to image heme proteins with high sensitivity. Photothermal microscopy, ${ }^{5-9}$ which relies on the detection of local heating generated by optical absorption of molecules, may overcome the difficulty of the poor sensitivity of direct absorption measurements. Photothermal microscopy is an emerging technique to detect absorbing microscopic objects, and is particularly suitable for imaging metal particles. ${ }^{7-9}$ Ultrafast spectroscopy experiments have shown that heme proteins have extremely fast internal conversion rates upon photoexcitation and associated ultrashort excited state lifetime $(<50 \mathrm{fs})$ of Soret band. ${ }^{3,4}$ Therefore, the accompanied high conversion efficiency (close to $100 \%$ ) from optical absorption to heat dissipation suggests that the photothermal effect could be an ideal contrast mechanism.

In all reported photothermal imaging schemes, onephoton linear absorption of the laser beam is used to induce local heating, which is then read out by a second probe beam. In contrast, we use two-photon nonlinear excitation by a near infrared (NIR) ultrafast laser as the heating source for heme proteins. This excitation scheme offers a number of

\footnotetext{
a) Author to whom correspondence should be addressed. Electronic mail: xie@ chemistry.harvard.edu.
}

different useful features. First, two photon absorption confines the absorption to the exact focal region instead of along the entire beam path, as a consequence of the nonlinear intensity dependence. Therefore, as shown in the inset of Fig. 1, compared to the conventional case using one-photon excitation, ${ }^{10}$ the resulting two-photon excited thermal gradient is less "dilute" and much more concentrated along the z-axis, which would enhance the readout signal generated by the probe beam. Second, compared to one-photon visible excitation, NIR light has much deeper penetration depth in scattering tissue and causes much less damage on biological samples. ${ }^{10}$ Third, the intense Soret band $(\sim 415 \mathrm{~nm})$ of heme proteins has recently been shown to exhibit a large and spe-

(a)

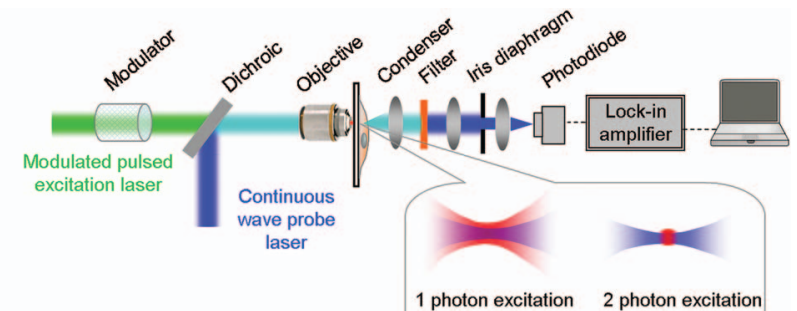

(b)

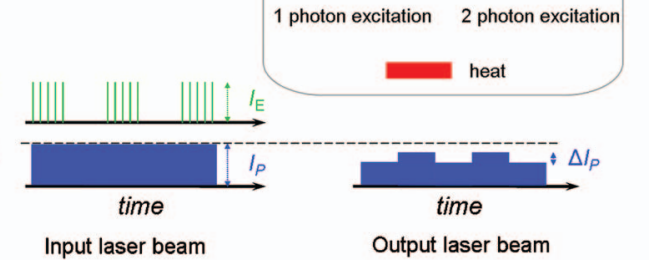

FIG. 1. (Color) Principle of two-photon excited photothermal lens microscopy. (a) Experimental apparatus. The incident ultrafast excitation $(\sim 200$ fs at $830 \mathrm{~nm}$ ) and continuous wave probe beams at $785 \mathrm{~nm}$ are spatially overlapped and focused onto the sample. A modulator switches the intensity of the excitation beam on-and-off at $\sim 100 \mathrm{kHz}$. While the collinear excitation and probe beams are raster scanned across the sample, the spectrally filtered probe beam is collected by the condenser-lens pair, descanned onto an iris diaphragm with adjustable position and aperture, and is refocused onto a large-area photodiode, and demodulated by a lock-in amplifier to create the image contrast. The inset shows that the thermal gradient (in red) generated by two-photon excitation is spatially more concentrated than that by onephoton excitation, especially along z-axis. (b) Principle of high-frequency modulation. As the intensity of the excitation beam is modulated on-and-off in time, the probe beam intensity on the detector is also modulated at the same frequency, when the iris diaphragm only passes the central portion of the probe beam. 

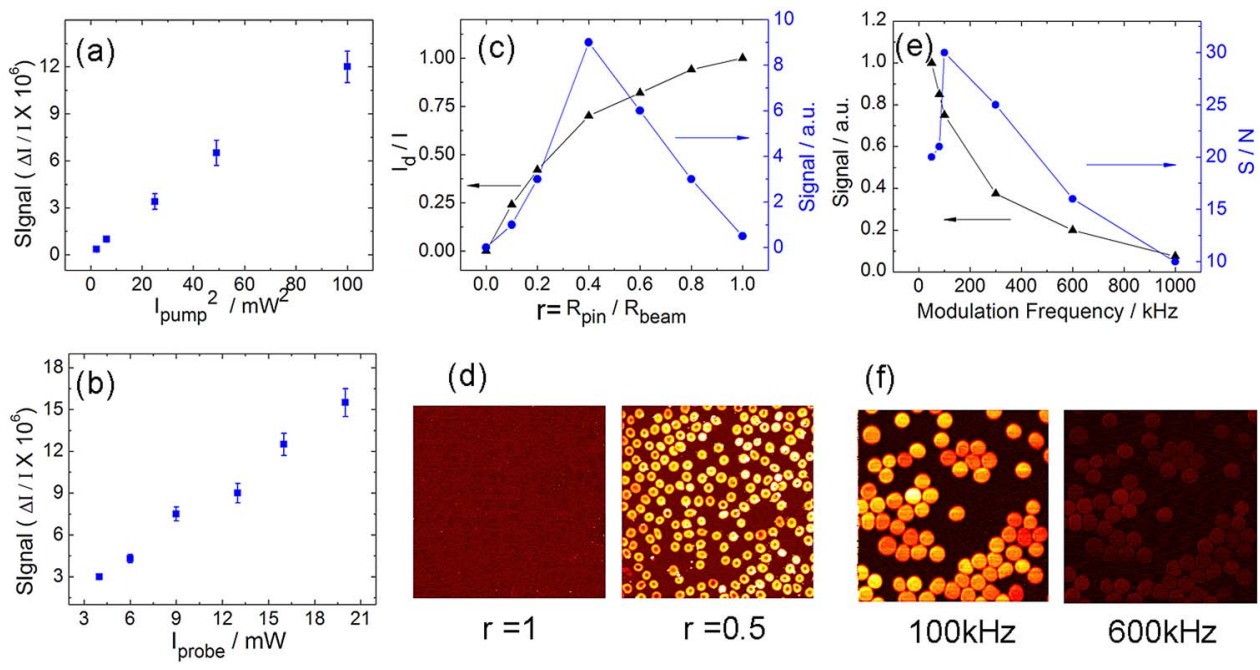

(d)

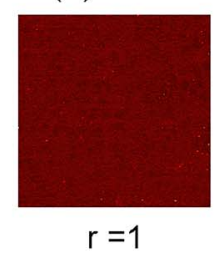

(f)

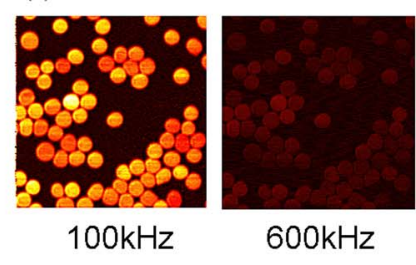

FIG. 2. (Color online) Characterization and optimization of imaging parameters. (a) Dependence of photothermal signal on the pump laser power in $100 \mu \mathrm{M}$ hemoglobin solution. (b) Dependence of photothermal signal on the probe laser power. (c) Dependence of photothermal signal and the detected probe intensity $\left(I_{d} / I\right)$ on the ratio $(r)$ of the iris radius $\left(R_{\text {pin }}\right)$ and the probe beam radius $\left(R_{\text {beam }}\right)$. (d) Photothermal image of individual red blood cells with $r=1$ and $r=0.5$. (e) Dependence of photothermal signal and signal-to-noise ratio on the modulation frequency from $50 \mathrm{kHz}$ to $1 \mathrm{MHz}$. To maintain high signal, low noise and reasonably fast imaging speed, the modulator is set at $\sim 100 \mathrm{kHz}$ for all of the following images.

cific two-photon absorption cross section near $830 \mathrm{~nm} .^{11}$ These desirable features of two-photon excited photothermal detection enable imaging heme proteins in living cells and in highly scattering tissues with superb sensitivity.

The schematic of laser scanning microscope is depicted in Fig. 1. A near IR laser beam at $830 \mathrm{~nm}$ with repetition rate of $76 \mathrm{MHz}$ pulse train ( 200 fs pulse width) serves as the excitation beam, while a stable continuous wave light at $785 \mathrm{~nm}$ serves as the probe beam. ${ }^{12}$ After collinearly combination by a dichroic mirror, these two beams are focused coaxially into the specimen by a microscope objective. The forward propagating beam is collected by a condenser. The probe beam is collected by the condenser-lens pair, spectrally cleaned by a filter, and focused onto a large-area Si photodiode.

A thermal lens detection scheme, which is among the most sensitive methods of the family of photothermal spectroscopy approaches, ${ }^{5,6}$ is adopted in our method to enhance the sensitivity of probe beam. We wish to detect the refractive index gradient generated by the excitation beam. To do so, an iris diaphragm with an adjustable aperture size and position is installed in front of the detector. Only the central portion of the probe beam instead of the entire beam is allowed to pass through the iris to hit the detector. The final two photon excited photothermal lens signal is dependent on $^{5}$

$$
S \propto \frac{I_{\mathrm{ex}}^{2} \cdot I_{\mathrm{pr}} \cdot \sigma_{2-\mathrm{p}} \cdot[c] \cdot \eta_{\mathrm{H}}}{\lambda_{\mathrm{pr}} \cdot \kappa}\left(\frac{d n}{d T}\right)_{\mathrm{P}},
$$

where $I_{\mathrm{ex}}$ and $I_{\mathrm{pr}}$ are the intensity of the excitation beam and probe beam, respectively, $\sigma_{2-p}$ is the two-photon absorption cross section, $[c]$ is the sample concentration, $\eta_{\mathrm{H}}$ is the yield of heat dissipation, $\lambda_{\mathrm{pr}}$ is the wavelength of the probe beam, $\kappa$ is the thermal conductivity, and $(d n / d T)_{\mathrm{P}}$ is the refractive index temperature coefficient at constant pressure.

To significantly reduce the detection noise, we implement a high-frequency $(>50 \mathrm{kHz})$ phase-sensitive detection scheme. In so doing, the probe laser intensity fluctuation, which primarily occurs at low frequency (dc to $10 \mathrm{kHz}$ ), can be circumvented, as has been previously applied in other spectroscopic and microscopic techniques. ${ }^{13,14}$ In the scheme shown in Fig. 1, the intensity of the excitation beam is modulated by an acoustic-optical modulator, creating a modulation of the photothermal signal at the same frequency. Such an induced modulation signal can then be sensitively extracted by a lock-in amplifier referenced to this modulation frequency. In this way, the photothermal signal is detected against a vanishing laser noise, offering superb sensitivity.

To verify the image contrast is indeed the result of the two-photon photothermal effect, we probe the photothermal signal of $100 \mu \mathrm{M}$ hemoglobin solution under different pump and probe laser power [Figs. 2(a) and 2(b)]. As is expected, the overall signal scales quadratically with the pump power and linearly with the probe power. We then demonstrate imaging individual red blood cells to optimize a few microscopic parameters. When the iris diaphragm is fully open to collect all the intensity of the forward propagating probe beam, the photothermal image, shown in Fig. 2(d), is essentially blank. However, when the iris is partially closed to block the peripheral portion of the probe beam, the corresponding image, shown in Fig. 2(d), clearly reveals individual red blood cells. The signal has a sensitive dependence on the collection aperture size [shown in Fig. 2(c)], which is a manifestation of the underlying thermal lens effect.

Modulation frequency of the phase sensitive detection is another crucial parameter in photothermal microscopy. As shown in Fig. 2(e), the absolute signal size increases as the modulation frequency is reduced because it takes time for the thermal gradient to buildup due to finite thermal conductivity. However, the laser noise of the probe beam also starts to increase for the slower modulation frequency. In addition, pixel dwell time has to be significantly longer than the modulation period to be able to demodulate reliably for each pixel. Therefore, based on the balancing between the signal size, the noise level, and the imaging speed, we choose $\sim 100 \mathrm{kHz}$ modulation frequency as the optimum for scanning microscopy. 

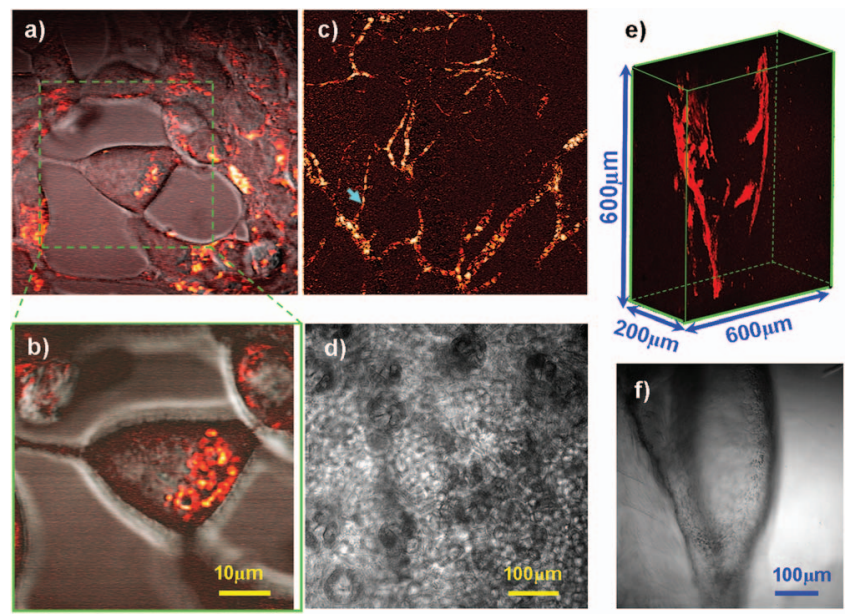

FIG. 3. (Color) Applications of two-photon excited photothermal lens microscopy in heme protein imaging. (a) Imaging distribution of cytochromes in live HEK-293 cells (red hot), overlapped with a transmission image of the probe beam (gray). (b) In a reduced volume scan of the indicated area in (a), individual mitochondria can be clearly resolved in this label-free manner. (c) Imaging the microvascular network in mouse ear tissue based on hemoglobin contrast. The image shows the blood vessel network surrounding sebaceous glands. Individual blood cells are shown to be lined up within a single capillary ( $<5 \mu \mathrm{m}$ in diameter). (d) Transmission image by the $\mathrm{cw}$ probe beam of the same tissue region as in (c). (e) 3D reconstruction of blood vessel network in zebrafish gill. (f) Transmission image of zebrafish gill by the $\mathrm{cw}$ probe beam. In all two-photon excited photothermal imaging experiments, the power of $200 \mathrm{fs} 830 \mathrm{~nm}$ excitation beam and $785 \mathrm{~nm} \mathrm{cw}$ probe beam are kept to be $\sim 10$ and $20 \mathrm{~mW}$, respectively, at the focus of the objective, with laser modulation frequency at $100 \mathrm{kHz}$ and pixel dwell time $\sim 200 \mu \mathrm{s}$

We then demonstrate the application of this microscopy in live cell imaging. Mitochondria contain membrane-bound cytochromes which have important roles in electron transport and controlling of apoptosis. Cytochromes are small protein molecules $(\sim 12 \mathrm{kD})$ and are difficult to label without affecting their normal physiology. They contain heme group as their key structural components, and therefore can be imaged in this label-free manner. As shown in Figs. 3(a) and 3(b), the asymmetric cellular distributions of organelles containing cytochromes can be clearly resolved. This technique could allow study of fusion-fission mitochondrial dynamics in live cells in a label-free manner. ${ }^{15}$

Finally, we demonstrate label-free imaging of microvascular network based on endogenous contrast from hemoglobin. Structure and hemodynamics of blood vessels play a major role in many biomedical processes such as angiogenesis in tumors. ${ }^{1}$ However, established techniques such as magnetic resonance imaging, computed tomography, positron emission tomography, ultrasound, and more recently, photoacoustic tomography, ${ }^{16}$ confocal, two-photon microscopy, ${ }^{17}$ and fluorescence microendoscopy ${ }^{18}$ either lack the spatial resolution needed to resolve individual red blood cells, or require exogenous contrast agents. ${ }^{1}$ Figure 3(c) shows the image of vascular network from a mouse ear. The capillaries are seen to wrap around the sebaceous glands shown in the transmission image [Fig. 3(d)]. Furthermore, we can also perform imaging on a whole organism level. Zebrafish, whose larvae are relatively transparent in early development, provides an ideal vertebrate model for cancer progression and angiogenesis and is readily amenable to ge- netic and pharmacological screening. ${ }^{19}$ Figure 3(e) shows a $3 \mathrm{D}$ reconstruction of the blood vessel of a larval zebrafish gill. In contrast with transmission image shown in Fig. 3(f), two-photon photothermal contrast allows deep penetration in scattering tissue.

From the perspective of instrumentation, this microscopy can be readily configured from a standard two-photon fluorescence microscope which is already equipped with the two-photon excitation source. The only additional elements required are a cw probe laser and a modulator/demodulator. We note that other femtosecond pump-probe techniques have recently been employed to image hemoglobin by using two synchronized femtosecond lasers, ${ }^{20}$ which is however technically complex and more expensive.

To summarize, two-photon excited photothermal lens microscopy enables label-free high-resolution imaging of heme protein in live cells, tissues, and organisms with intrinsic 3D optical sectioning and high sensitivity. The technique opens up further possibilities of functional imaging of heme proteins such as the oxygenation level of hemoglobin and redox state dynamics of cytochromes both in cellular and in tissue/organism levels.

We thank Christian Freudiger and Brian Saar for helpful discussions; Harvard MCB Mouse facility for providing euthanized mice, and Harvard MCB zebrafish facility for providing euthanized zebrafish. S.L. and W.M. contribute equally to this work. This work was supported by National Science Foundation (Grant No. DBI-0649892) and U.S Department of Energy's Basic Energy Sciences Program (Grant No. DE-FG02-07ER15875).

${ }^{1}$ D. M. McDonald and P. L. Choyke, Nat. Med. 9, 713 (2003)

${ }^{2}$ X. Jiang and X. Wang, Annu. Rev. Biochem. 73, 87 (2004).

${ }^{3}$ P. M. Champion and G. J. Perreault, J. Chem. Phys. 75, 490 (1981).

${ }^{4}$ R. Jimenez and F. E. Romesberg, J. Phys. Chem. B 106, 9172 (2002).

${ }^{5}$ S. E. Bialkowski, Photothermal Spectroscopy Methods for Chemical Analysis (Wiley, New York, 1996).

${ }^{6}$ M. Tokeshi, M. Uchida, A. Hibara, T. Sawada, and T. Kitamori, Anal. Chem. 73, 2112 (2001)

${ }^{7}$ D. Boyer, P. Tamarat, A. Maali, B. Lounis, and M. Orrit, Science $\mathbf{2 9 7}$ 1160 (2002)

${ }^{8}$ L. Cognet, C. Tardin, D. Boyer, D. Choquet, P. Tamarat, and B. Lounis, Proc. Natl. Acad. Sci. U.S.A. 100, 11350 (2003).

${ }^{9}$ A. V. Brusnichkin, D. A. Nedosekin, M. A. Proskurnin, and V. P. Zharov, Appl. Spectrosc. 61, 1191 (2007).

${ }^{10}$ W. Denk, J. H. Strickler, and W. W. Webb, Science 248, 73 (1990).

${ }^{11}$ G. O. Clay, C. B. Schaffer, and D. Kleinfeld, J. Chem. Phys. 126, 025102 (2007).

${ }^{12}$ See supplementary material at http://dx.doi.org/10.1063/1.3308485 for detailed experimental setups and sample preparations.

${ }^{13}$ C. W. Freudiger, W. Min, B. G. Saar, S. Lu, G. R. Holtom, C. He, J. C. Tsai, J. X. Kang, and X. S. Xie, Science 322, 1857 (2008).

${ }^{14}$ W. Min, S. Lu, S. Chong, R. Roy, G. R. Holtom, and X. S. Xie, Nature (London) 461, 1105 (2009).

${ }^{15}$ S. A. Detmer and D. C. Chan, Nat. Rev. Mol. Cell Biol. 8, 870 (2007).

${ }^{16}$ E. W. Stein, K. Maslov, and L. V. Wang, J. Biomed. Opt. 14, 020502 (2009).

${ }^{17}$ C. B. Schaffer, B. Friedman, N. Nishimura, L. F. Schroeder, P. S. Tsai, F. F. Ebner, P. D. Lyden, and D. Kleinfeld, PLoS Biol. 4, e22 (2006).

${ }^{18}$ A. Monfared, N. H. Blevins, E. L. Cheung, J. C. Jung, G. Popelka, and M. J. Schnitzer, Otol. Neurotol. 27, 144 (2006).

${ }^{19}$ K. Stoletov, V. Montel, R. D. Lester, S. L. Gonias, and R. Klemke, Proc. Natl. Acad. Sci. U.S.A. 104, 17406 (2007).

${ }^{20}$ D. Fu, T. Ye, T. E. Matthews, B. J. Chen, G. Yurtserver, and W. S. Warren, Opt. Lett. 32, 2641 (2007). 